\begin{tabular}{|c|c|c|c|c|} 
Revista Praxis & ISSN: $1657-4915$ & Vol. 12 & $90-102$ & Enero - Diciembre de 2016 \\
\hline
\end{tabular}

\title{
LA FORMACIÓN DOCENTE EN LOS PROCESOS DE MEDIACIÓN DIDÁCTICA
}

\author{
TEACHER EDUCATION IN THE PROCESS OF TEACHING MEDIATION
}

Edgar Andrés Espinosa-Ríos ${ }^{1}$

\begin{abstract}
RESUMEN
El artículo pretende mostrar una reflexión conceptual sobre la importancia de la formación docente, el cual surge a partir de la investigación y revisión de diferentes fuentes bibliográficas. Dicha revisión es de índole inductiva, en él se tiene en cuenta el análisis de contenido planteado por Abela (s.f.), estableciendo así la unidad de contexto, las unidades de muestreo y las diferentes categorías tales como: la relación entre la teoría y la práctica, la importancia de la formación docente en los procesos de mediación didáctica, la teoría de Vygotsky en los procesos de mediación didáctica, la mediación en los procesos de enseñanza-aprendizaje, la formación docente en los procesos de mediación didáctica, entre otras, dichas categorías orientan la reflexión del presente documento. Para ello se tiene en cuenta los aportes que realizan autores como Tébar, Schön y Vygotsky entre otros.

Se brindan aportes frente a la manera de mejorar los procesos de mediación didáctica para la enseñanza de las Ciencias Naturales en los docentes en formación. En él se recalca la importancia de la formación disciplinar, pedagógica, didáctica del docente en formación para la enseñanza de la disciplina, como a su vez su capacidad de reflexión y su importancia en los procesos de mediación didáctica.
\end{abstract}

Palabras clave: Mediación didáctica, conocimiento científico escolar, formación docente.

\section{ABSTRACT}

The article shows a conceptual reflection on the importance of teacher training, which arises from the research and review of various literature sources. This review is from inductive nature, it takes into account the content analysis proposed by Abela (sf), establishing the context unit, the sampling units and different categories such as: the relationship between theory and practice the importance of teacher training in educational mediation processes, Vygotsky's theory in the process of educational mediation, mediation in the process of teaching - teacher training in educational mediation processes, among others, those categories oriented reflection of this document. To do this we take into account the contributions made by authors like Tébar, Schön and Vygotsky among others.

Fecha de recepción: Agosto 03 de 2015 Fecha de aceptación: Marzo 31 de 2016

Tipología: Artículo de Reflexión

Para citar este artículo: Espinosa, R. E. (2016). La formación docente en los procesos de mediación didáctica. Praxis. Vol. 12,90 - 102

1. Docente de la Universidad del Valle. Magister en la Enseñanza de las Ciencias de la Universidad del Valle. Colombia. Email: edane2009@hotmail.com andres.espinosa@correounivalle.edu.co 
Contributions are provided in front of the way to improve the processes of teaching mediation for the teaching of natural sciences in teacher trainees. It highlights the importance of training discipline, pedagogical, didactic teacher training for teaching discipline, such as turn their capacity for reflection and its importance in the processes of educational mediation is emphasized.

Keywords: Teaching mediation, school scientific knowledge, teacher training

\section{INTRODUCCIÓN}

$\mathrm{E}^{\mathrm{l}}$ propósito del artículo es brindar algunos elementos que permitan mejorar los procesos de mediación didáctica para la enseñanza de las Ciencias Naturales en los docentes en formación, dichos elementos son construidos a partir de la investigación y revisión de diferentes fuentes bibliográficas, de tal manera que permita mostrar la importancia de la reflexión por parte del docente frente a los procesos de mediación didáctica.

Teniendo en cuenta el propósito del artículo se hace necesario tener claro que la escuela como parte de una sociedad se caracteriza porque en ella se hace de manera formal la construcción de un conocimiento científico escolar, el cual es el resultado de la interacción entre el conocimiento del docente y el del estudiante (Zambrano, 2000). Para facilitar la construcción del conocimiento científico escolar es necesario la implementación de diferentes materiales y estrategias didácticas tales como las prácticas de laboratorio, los textos escolares, las TIC, entre otras, que bien empleadas en el aula de clase facilitaran dicha construcción.

En los procesos de construcción del conocimiento científico escolar llevado a cabo en el aula se reconocen tres agentes que participan de forma directa: El docente, el estudiante y el contenido de las ciencias el cual debe ser representado y formulado a través de los diferentes materiales y estrategias didácticas, la interacción entre los tres agentes se materializa en el acto educativo es decir, la acción que se lleva en el aula de clase la cual debe conllevar a la construcción del conocimiento científico escolar.

El docente se caracteriza por ser aquella persona que tiene un cumulo de experiencia y conocimientos que ha sido el resultado de su desarrollo por el paso de diferentes instituciones educativas, el estudiante se convierte en aquella persona con un cumulo de conocimientos, pero que han sido el resultado en su gran mayoría por las experiencias adquiridas en su diario vivir y el paso por algunas instituciones formales y no formales y el contenido de las ciencias, el cual es representado y formulado a través de los materiales didácticos que utiliza el docente y facilita la "mediación didáctica" entre ese conocimiento del docente y el conocimiento del estudiante, es decir, la relación entre el decir y el hacer, entre la teoría y la práctica, propiciando así la construcción de un conocimiento científico escolar.

Siendo los diferentes materiales didácticos, las herramientas y las estrategias que se implementan en el aula de gran importancia en los procesos de construcción del conocimiento, se hace relevante indagar sobre las dificultades que se han presentado al respecto, entre ellas se puede mencionar que en muchos de los casos no se promueve el desarrollo de un pensamiento crítico por parte de los estudiantes, solo facilitan el comprender y aprender una teoría, en otros casos dichas estrategias y herramientas empleadas no acercan a los estudiantes al contexto en el cual se encuentran, lo cual no favorece la construcción del conocimiento. A partir de lo anterior se hace necesario revisar los planteamientos de diversos autores tales como como Serrano (2010), Korthagen (2010), Seré (2002) frente al uso de las prácticas de laboratorio; como a su vez los planteamientos realizados por Vaillant (2013), y el Ministerio de Educación Nacional (2008) frente al uso e implementación de las TIC en el aula entre otros. Como consecuencia de lo anterior y en razón de las diferentes dificultades encontradas en los procesos llevados en el aula, surge la necesidad de indagar sobre los procesos 
de formación docente, de ahí la importancia de los planteamientos realizados por el Ministerio de Educación Nacional (2012), Briceño (2012), Schön (1987).

\section{METODOLOGÍA}

La metodología desarrollada como parte de la investigación y revisión bibliográfica busca brindar elementos que permitan mejorar los procesos de mediación didáctica para la enseñanza de las ciencias naturales en los docentes en formación. Para lograr lo anterior se tuvo en cuenta la técnica de análisis de contenido planteado por Abela (s.f.) por medio de la cual se establece la unidad de contexto denominada la formación docente en los procesos de mediación didáctica, a partir del mismo se establecen las siguientes unidades de muestreo: La relación entre la teoría y la práctica, las TIC como una necesidad dentro de la formación docente y la importancia de la reflexión en los procesos de formación docente. Posteriormente surgen las siguientes categorías las cuales son el fruto de la reflexión en el documento: la relación entre la teoría y la práctica, la importancia de la formación docente en los procesos de mediación didáctica, la teoría de Vygotsky en los procesos de mediación didáctica, la mediación en los procesos de enseñanza-aprendizaje, la formación docente en los procesos de mediación didáctica, la importancia de la reflexión en los procesos de formación, la reflexión a partir de la enseñanza de las ciencias, la reflexión sobre sí mismos, como docentes en formación, reflexionar sobre la opinión de los expertos, reflexionar sobre su propia enseñanza, la mediación didáctica en el aula de clase, las actividades de aprendizaje como parte de la mediación didáctica. A partir de lo anterior se establecen las conclusiones pertinentes.

\section{La relación entre la teoría y la práctica}

A través de la historia se ha tratado de explicar el porqué de la separación de la relación teoríapráctica, encontrando dos posiciones que se han enfrentado entre sí, la primera hace referencia al enfoque científico-tecnológico y la segunda al enfoque hermenéutico-interpretativo. El primer caso hace referencia al poder de la teoría para sobreponerse a la práctica en donde el docente es visualizado como aquel que tiene el conocimiento absoluto y es a partir de dicho conocimiento que se construye la práctica y en el segundo la práctica para dominar a la teoría, en donde el docente se concibe como un artesano que construye el conocimiento acumulado a partir de sus experiencias y mejorando la practica a partir de la acción, valorándola por encima del conocimiento científico (Böhm, citado por Álvarez, 2012).

Clark \& Yinger, Sjavelson (1998) \& Stern (1989) (citados por Serrano, 2010) reconocen que el docente dentro de su proceso de formación construye y fortalece básicamente dos tipos de conocimientos, el conocimiento disciplinar y el conocimiento pedagógico, además del poseer una capacidad de reflexión, de tomar decisiones, de emitir juicios y de generar rutinas que conllevan a su desarrollo y guían su actuar. Wittrock (1989) planteaba que existen muchas variables en los procesos de enseñanza-aprendizaje y que dichas variables tales como la materia, el grado, número de estudiantes en el aula de clase, serian la esencia misma del modelo de enseñanza del docente y determinan en gran medida el comportamiento del mismo.

A partir de lo anterior el docente construye un modelo de pensamiento, el cual se materializa en el aula de clase. Estas premisas mencionadas anteriormente conllevaron a cambiar la concepción que en muchas ocasiones se tenía del docente, las cuales respondían al enfoque conductista de la enseñanza, pasando a un enfoque constructivista, en el cual construye, elabora y comprueba su teoría a partir del mundo que lo rodea (Clark, citado por Serrano, 2010).

Wittrock (1989) manifiesta que muchas investigaciones comenzaron a centrarse en aspectos menos visibles por parte del docente, aspectos que estaban más asociados a las formas de pensamiento, de emitir juicios o tomar decisiones, por lo tanto, si se deseaba conocer de forma adecuada las elecciones que los docentes hacen en el aula de clase y los fundamentos que tienen al momento de tomar decisiones, de 
emitir juicios respecto a los estudiantes, a los procesos cognitivos a partir de los cuales pueden encadenar todas las acciones que lleva en el aula de clase, es necesario tener presente los procesos de pensamiento del docente antes, durante y después de la enseñanza; por ejemplo, los procesos cognitivos del docente observados en el transcurso de su planificación, muestran que emplearon varios métodos tales como, emplear técnicas donde el docente exprese el pensamiento en voz alta, utilizando materiales reales del docente o el cuaderno de planificación o los materiales preparados por los investigadores; los resultados obtenidos muestran que la mayoría de los docentes al planificar se centraban más en el contenido y las actividades que en los objetivos formales y las características de los estudiantes.

El cambio de concepción de pensamiento del docente fue guiado por los principios de los modelos de toma de decisiones y del modelo de procesamiento de la información. El primer modelo concibe al docente como aquella persona que está valorando las situaciones, procesa la información sobre dichas situaciones y a partir de ahí toma decisiones sobre qué hacer, guía las acciones y a partir de las decisiones tomadas observa los efectos sobre las acciones de los estudiantes. El segundo modelo (procesamiento de la información) concibe al docente como una persona que es capaz de enfrentarse a un ambiente de tareas que resulta ser muy complejo, en el cual el docente tiene la capacidad de atender a un número en particular de aspectos del ambiente e ignorar el resto.

Estas transformaciones sobre las concepciones de pensamiento del docente conllevaron a generar varios cambios en la forma de investigar sobre las concepciones de aprendizaje, la cual se abordan de modos diferentes, por ejemplo, surgen los estudios del metaconocimiento, los cuales están dirigidos al conocimiento que manifiestan los profesores sobre las condiciones del estudiante y las tareas de aprendizaje y sobre las estrategias de aprendizaje Flavell (citado por Serrano, 2010). Aparece el enfoque fenomenológico, el cual indaga en las diferentes formas en que los docentes interpretan su propio aprendizaje.
Otros autores como Korthagen (2010) han realizado investigaciones frente a la ruptura entre la teoría y la práctica. El autor plantea que las principales causas son la resistencia del profesorado a cambiar la forma de pensar, al poco contacto con el conocimiento pedagógico, al lenguaje empleado en el aula de clase, que en muchas ocasiones es muy formal, a la poca relación entre el conocimiento disciplinar y los contextos escolares. A su vez el autor plantea que para mejorar dicha relación es necesario fortalecer la formación de los docentes y la capacidad de reflexión frente a sus procesos de enseñanza-aprendizaje.

La ruptura entre la teoría y la práctica se hace más visible al momento de implementar por parte de los docentes los diferentes materiales didácticos en el aula de clase. Son muchos los autores que han realizado investigaciones frente a ello, por lo cual se muestran algunos resultados obtenidos en la implementación de aquellos materiales didácticos que se emplean con mucha frecuencia:

Briceño, González \& Mosquera (2012) manifiestan que las prácticas de laboratorio no han contribuido al desarrollo de ideas científicas en los estudiantes porque al momento de implementarlas los docentes lo hacen como una forma de verificar un conocimiento ya existente en los libros de texto. Otros autores como Seré (2002) muestran que muchos docentes al implementar las prácticas de laboratorio no "invitan" a los estudiantes a reflexionar sobre la teoría aprendida, solo los lleva a comprender y aprender una teoría; es decir la efectividad de ellas en cuanto a la construcción del conocimiento científico escolar no radica en la herramienta misma, está supeditada en gran medida a la forma y el momento en el cual el docente las emplea en los procesos de mediación.

Vaillant (2013) realizó una investigación denominada la integración de TIC en los sistemas de formación docente inicial y continúa para la educación básica en américa latina avalado por la Unicef en la cual plantea varios puntos tales como: 
- Aunque las TIC pueden estar inmersas en las instituciones educativas, ello no asegura su uso efectivo por parte de los docentes, por eso es necesario la formación continua y su integración a la formación inicial docente, lo anterior está estrechamente vinculado a la actitud y al interés de utilizarlo por parte de los mismos en los procesos de mediación en el aula de clase.

- La implementación de las TIC en el aula de clase, se hace con mayor eficacia si los maestros han sido formados desde el inicio en este campo, lo que la autora denomina formación inicial y continua del docente, ya que no solo se debe pensar en el desarrollo de habilidades en TIC, sino en los métodos en los que se prepara al maestro o al docente para la integración de las tecnologías en el aula de clase.

El MEN (2008) en el Programa Nacional de Innovación Educativa con Uso de TIC plantea el deseo de formar a los docentes en TIC entre otras cosas, como una necesidad de implementar estos materiales didácticos en el aula de clase, para lo cual es necesario el desarrollo de competencias teniendo bien claro que antes del desarrollo de las competencias y la implementación de cualquier material didáctico en los procesos de mediación en el aula de clase, se debe promover el uso crítico desde actitudes positivas hacia la comunicación, colaboración y construcción del conocimiento.

\section{La importancia de la formación docente en los procesos de mediación didáctica}

Tébar (2009) plantea que la mediación didáctica posee como principio fundamental la creencia en la potenciación y la perfectibilidad de todo ser humano. De ahí la importancia de entender la mediación como una posición humanizante y positiva, de tal forma que potencialice la relación del individuo con su entorno. Teniendo en cuenta lo anterior la mediación tiene como principal objetivo el desarrollo de habilidades en el estudiante lo que debe conllevar al desarrollo de una autonomía plena. Autores como Martínez (2013) plantea que la autonomía se puede alcanzar en los sujetos en la medida en que se propicie la seguridad, la confianza, el compromiso y la metacognición acompañados de estilos de vida saludables a partir de la implementación de estrategias pedagógicas que promuevan la motivación y el desarrollo de diferentes habilidades. Como base de esta construcción dinámica se encuentra el concepto de desarrollo potencial planteado por Vigotsky en 1995.

Es bien entendido que muchos materiales inicialmente no fueron diseñados para ser implementados en la escuela, pero también es conocido que ellos se pueden implementar y se pueden convertir en un material clave para mejorar los procesos de enseñanza y aprendizaje, de tal forma que se potencialicen las habilidades de los estudiantes. Para lograr lo anterior es necesario tener en cuenta varios factores, de tal manera que la implementación de ellos en la escuela no se convierta en una simple moda social sin trascendencia; por ejemplo, es necesario que se le dé la debida importancia desde lo pedagógico y didáctico de tal forma que se pueda potencializar al máximo su implementación. Para potencializar dichas características el docente debe reflexionar sobre ellos y el papel que jugará en dicho proceso educativo. Se debe tener muy claro que el material didáctico por si solo no garantiza nada, y solo adquiere gran valor cuando es el docente quien muestra su necesidad en el aula de clase (a partir del conocimiento pedagógico y disciplinar que el docente posee), direccionándolo al objetivo deseado, de tal forma que favorezca los procesos de mediación didáctica (Espinosa, 2009).

Teniendo en cuenta lo anterior se puede inferir que un papel importante en los procesos de mediación didáctica, lo juega la formación que presentan los docentes, pues son éstas las que orientan el manejo de las mismas en el aula de clase, es por ello que se hace necesario plantear que los procesos de formación docente deben tener presente una planeación previa provista de un saber académico (saber qué), el cual es un conjunto de concepciones resultados de su formación, y su experiencia; de la práctica (saber hacer) y de los valores (saber ser) los cuales hacen referencia al ser como individuo (Ministerio de Tecnologías de la Información y las Comunicaciones, 2012). 
Lo anterior hace énfasis en dos ejes fundamentales, el primero el conocimiento académico (conocimiento propio del docente o conocimiento profesional) y el segundo a la aplicación de dicho conocimiento (saber hacer), es por ello que deben establecer una simbiosis de tal manera que le permita al docente analizar los diferentes problemas que se le vayan presentando para así darles solución, dicha simbiosis se podrá realizar con mayor facilidad cuando el docente los comienza a vivenciar desde lo práctico, de esta manera podrá ir desarrollando conceptualmente sus ideas y conocimientos (MEN, 2012).

Esta constante construcción de su conocimiento tendrá gran significancia para el docente en la medida en que lo haga consciente y tangible (Korthagen, 2010), si el docente reflexiona continuamente: sobre la enseñanza llevada a cabo por otros, sobre su propia enseñanza, sobre sí mismo como aprendiz de ciencias a través de actividades científicas, sobre las opiniones de los expertos acerca de la enseñanza a través de diferentes lecturas a partir de sus actividades sobre sus saberes, concebirá ésta última de forma dinámica, convirtiéndose en un sistema formativo en la cual diariamente va reconstruyendo su práctica (Abell \& Bryan, citados por Candela, 2012). Lo anterior se justifica porque existe una gran tendencia por parte de los docentes a volverse repetitivos en su quehacer como docente y por ende a no percatarse de las dificultades en su enseñanza (Mellado, 2003). Teniendo en cuenta lo anterior se debe entender que estos procesos no son inmediatistas, son procesos que se dan paulatinamente y en la medida en que los docentes reflexionen constantemente sobre su quehacer diario se mejorara los procesos de enseñanza y aprendizaje en el aula de clase.

\section{La Teoría de Vygotsky en los Procesos de Mediación Didáctica}

Vygotsky centra sus estudios en establecer la relación existente entre el objeto de conocimiento y el sujeto, lo anterior lo logra a partir del planteamiento interaccionista dialéctico objetosujeto $(\mathrm{O}-\mathrm{S})$, en la cual hay una relación de recíproca influencia, a dicha relación autores como Yaroshevsky (citado por Hernández, 1997) la denominan actividad objetual.

En los procesos de enseñanza y aprendizaje se establece una relación entre el sujeto y el objeto de conocimiento, esta relación está mediada por la actividad que el sujeto realiza sobre el objeto de conocimiento, para ello se vale de diferentes instrumentos socioculturales, dichos instrumentos Vygotsky las denomina herramientas y signos (ver figura 1), la primera (herramienta) orienta la actividad del sujeto y la segunda (el signo) genera cambios en el sujeto que efectúa dicha actividad al interior del mismo.(Hernández, 1997)

Figura 1.

Esquema de las relaciones entre sujeto y objeto en la psicología desde Vygotsky

Herramientas

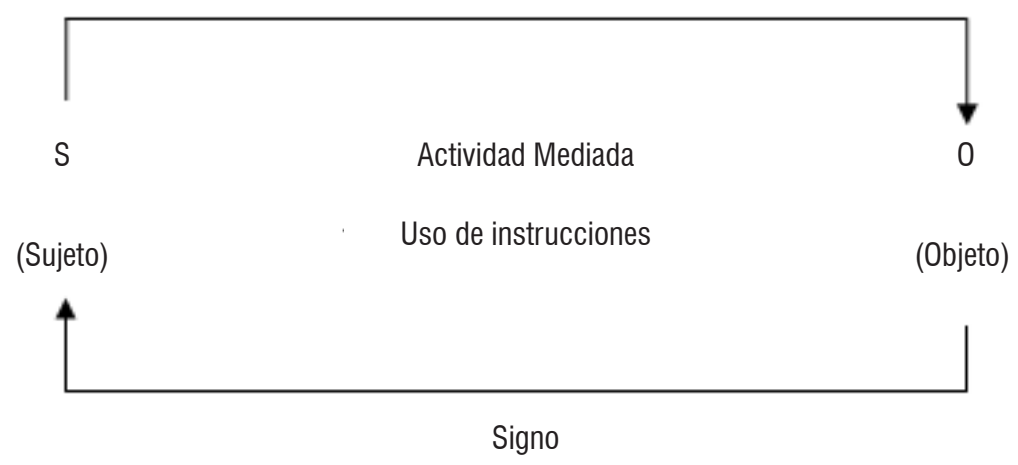

Fuente: Hernández, (1997) 


\section{¿Qué se entiende por signo?}

El concepto de signo planteado por Vygotsky como elemento fundamental en la mediación de los procesos de enseñanza-aprendizaje se mira desde dos perspectivas, la primera se plantea como un estímulo intermedio dentro de dicho proceso, y la segunda como instrumento que media socialmente. El signo es un momento que se da entre el estímulo inicial y la respuesta a dicho estímulo, siendo la interpretación del signo dada por el sujeto la que determina el estímulo siguiente (Gutiérrez, Ball \& Marquez, 2007). Para el caso de los humanos, Vygotsky asume una condición activa en el momento de interpretar las diferentes señales, lo cual le da una característica diferencial a las demás especies animales. Estas señales para los seres humanos se convierten en signos a la hora en que no se remiten a ser más señales no naturales "aprehendidas por la experiencia sino por señales no naturales, inventadas intencionadamente" (Gutiérrez et al., 2007) No se trata de señales sino de signos, a los que Vygotsky define como "estímulos-medio artificiales introducidos por el hombre". Perinat (1986, citado por Gutiérrez et al., 2007) plantea que de este modo los signos son herramientas creadas por las personas para regular su propio comportamiento y el de las demás, por lo cual todo signo implica por parte del receptor una elaboración cognitiva de dimensiones complejas para lo cual se asume por parte del receptor una interpretación o atribución de significado, es decir, para poder entender un signo se debe tener en cuenta otro(s) signo(s) que me permitan la interiorización del nuevo signo.

El signo como mediador social se emplea como una herramienta que tiene como fin establecer una comunicación a través de las relaciones sociales; es decir, el signo se convertirá en un instrumento para mediar en el comportamiento, las creencias y las percepciones del otro, en primera instancia; posteriormente, el signo transformará al sujeto en sí mismo, lo que implica una interiorización del mismo. Como el signo siempre está dirigido al individuo, este le pertenecerá tanto a quien lo produce como a quien lo recibe, $y$ tratará sobre algo, este representará a otra cosa, objeto, persona o evento en particular, tal como lo plantea Sisto (1998, citado por Gutiérrez et al., 2007).

Si el signo, como vuelvo a repetir, es algo que representa a otro algo para alguien, entonces el signo sólo es tal cuando una expresión y un contenido están en correlación, y ambos elementos se han convertido en una unidad que emerge de esa correlación. Así el signo no es una entidad física, ni tampoco una entidad semiótica fija. El signo no es el lugar de encuentro de elementos mutuamente independientes. Por ello se puede manifestar que no existen signos, sino funciones semióticas. De ahí que Vygotsky haya escogido como unidad de estudio de la conciencia no al signo, entendido como la palabra, sino como el significado de la palabra. (Gutiérrez et al., 2007, p.3)

\section{La Mediación en los Procesos de Enseñanza-Aprendizaje}

La mediación concebida dentro de los procesos de enseñanza-aprendizaje se debe interpretar como todas aquellas acciones que se realicen en beneficio de los procesos de la enseñanza y el aprendizaje, dichas mediaciones se verán representadas por la actividad, la intervención y el material didáctico que se implemente en el proceso. La intervención busca en primera instancia la comunicación entre los estudiantes y el mediador, de tal manera que favorezca el razonamiento y la comprensión de las ideas a través de los sentidos. Fainholc (2004) plantea que dicha intervención debe estar enmarcada en una educación que fomente la participación, la creatividad, la expresividad, la racionalidad, de tal forma que potencialice las habilidades que se tienen tal como lo enuncia Vigotsky en sus planteamientos sobre la zona de desarrollo próximo.

La implementación de los materiales didácticos en los procesos de mediación pueden desarrollar y potencializar las habilidades mencionadas pues ellas facilitaran un dialogo y una interactividad entre el individuo y el conocimiento; según Gadamer (citado por Fainholc, 2004) destaca que el trabajo interpretativo brinda elementos sobre 
el "dialogo" en la medida en que el individuo a partir de la interpretación establece un vínculo a través de preguntas y respuestas que conllevaran a un entendimiento.

\section{La Formación Docente en los Procesos de Mediación Didáctica}

Es muy difícil establecer con total certeza cuales son todas las cualidades especificas del docente de tal forma que permitan establecer una relación pura entre él y los materiales didácticos, pero si se pueden proponer algunas cualidades que hagan está relación más duradera, por ejemplo, la primera y fundamental es la disposición del docente al cambio, la segunda es generar conciencia a que la práctica educativa invita a una constante reflexión frente a la enseñanza y su papel activo en ella, a la forma como se debe concebir al estudiante dentro de ese proceso, al dominio de su conocimiento disciplinar y por ende a la implementación de diferentes materiales didácticos (Briceño et al., 2012).

Es fundamental que el docente no solamente se conforme con un buen dominio del tema o simplemente el buen conocimiento de materiales didácticos, su condición debe trascender más allá del simple echo del conocimiento mismo, debe hacerse cuestionamientos tales como la forma como se debe enseñar, como estoy enseñando y como los estudiantes aprenden; lo anterior implica que el docente desarrolle ciertas habilidades o competencias que deben trascender del mero hecho técnico y tecnológico, pues no son ellas únicamente el factor que permitirá mejorar los procesos de enseñanza y aprendizaje, se debe pensar en habilidades cognitivas pues es el docente quien a partir de sus concepciones y el reflejo de estos en el aula de clase son los que contribuirán a mejorar los procesos de enseñanza y aprendizaje. Teniendo en cuenta lo anterior y según lo planteado por el MEN (2012) un docente se debe caracterizar por tener un manejo disciplinar (manejo del conocimiento científico), su carácter investigativo (entendido como un proceso en donde confluyen la teoría y la práctica la cual implica un pensamiento reflexivo y metodológico), la pedagogía y su actitud frente al quehacer pedagógico (vital para su quehacer en el aula de clase), su capacidad comunicativa (se busca una comunicación efectiva, multidireccional, reflexiva, participativa y no centrada exclusivamente en el docente, fundamental en el aprendizaje en los estudiantes), los avances técnicos y tecnológicos (manejo y uso de todas las herramientas que se circunscriban en las TIC) y su capacidad evaluativa (proceso que no puede ser enfrentado como una cifra, o un número, es una oportunidad de mejorar y de formar).

\section{La Importancia De La Reflexión En Los Procesos De Formación}

Las investigaciones en educación deben estar encaminadas en cambiar la estructura de conocimiento, creencias y valores sobre la enseñanza y aprendizaje de las ciencias con los cuales llegan los docentes en formación a los diferentes programas, además los propósitos que los diferentes programas de educación se plantean generalmente no son alcanzados a cabalidad por todos los docentes, es decir, son pocos los que logran cambiar su estructura conceptual, de ahí que las diferentes experiencias que tienen los docentes en formación durante las etapas de su vida académica toman gran relevancia en los momentos en que inician su período de aprendizaje sobre el cómo enseñar.

Teniendo en cuenta lo anterior, los formadores de formadores deberían diseñar programas que permitan preparar e inducir el desarrollo profesional, de tal manera que se generen ambientes de aprendizajes que permitan a los futuros docentes evolucionar su estructura conceptual, con marcos conceptuales más coherentes y desde una perspectiva constructivista social. A partir de lo anterior, el rol del docente en la formación de los futuros docentes juega un papel crucial, pues son estos los encargados de modelar las creencias y valores sobre la enseñanza y aprendizaje de esta disciplina con la que los docentes en formación llegan a los programas de educación, teniendo como referente los marcos filosóficos constructivistas sociales (Abell \& Bryan, 1997).

Desde este punto de vista es necesario que los programas de formación, inducción y desarrollo del docente en ciencias presenten como pilar en 
su estructura educativa una orientación reflexiva, pues ella establece que aprender a enseñar ciencias es similar a aprender ciencias por uno mismo en la medida en que es un proceso en el cual el docente en formación re-evalúa y replantea sus propias teorías a partir de situaciones particulares, teniendo como orientador al docente que contribuye en su formación. Este tipo de orientación facilita que el docente en formación e incluso aquellos docentes en ejercicio hagan explicitas sus ideas, creencias y valores sobre la forma de enseñar y aprender ciencias, además da la oportunidad de generar experiencias prácticas que les da claridad en gran medida frente a las teorías personales de tal forma que ellas puedan evolucionar (Abell \& Bryan, 1997).

Autores como Schön (1987) también plantea la necesidad de reflexionar del docente sobre su quehacer diario, pues en la medida en que genere conciencia frente a la importancia de su papel en los procesos de mediación didáctica y la necesidad de relacionar la teoría y la práctica favorecerán los procesos de enseñanza y aprendizaje. Sumado a lo anterior Martínez (2013) afirma que al reflexionar sobre aquellas prácticas que se realizan de forma inconsciente se genera un espacio para las teorizaciones pedagógicas permitiendo así la comprensión del acto educativo y el fortalecimiento del saber pedagógico.

Schön (1987) plantea una epistemología de la práctica, la cual surge a partir de observar la crisis de las diferentes profesiones, pues manifiesta que el conocimiento que se construye a partir de la racionalidad y es adquirido en la educación formal, no responde de forma inmediata a los problemas prácticos que se puedan presentar en diferentes situaciones, por ello el autor plantea la necesidad de cambiar la estructura conceptual del docente, de tal forma que le permita tomar decisiones inmediatas para posteriormente invitarlo a reflexionar sobre las acciones tomadas en los diferentes momentos.

A este conocimiento que se coloca en práctica o en situaciones inmediatas le es llamado "el conocimiento en acción” y se considera un saber que no proviene de una previa operación intelectual, es fruto de una necesidad inmediata que conlleva de igual forma una acción, es un conocimiento que en ocasiones cuesta trabajo manifestarlo al igual que describirlo, es un conocimiento "tácito” que en ocasiones es llamado por muchas personas sentido común (Schön, 1987).

Frente a la reflexión en acción, Vasco (citado por López \& Basto, 2010) plantea cinco aforismos que permiten comprender el origen de la reflexión y la práctica del docente reflexivo: “... los sistemas de acciones se van decantando en prácticas; los fracasos de las prácticas llevan a la reflexión. Nace la praxis; la praxis se transforma en virtud de esa reflexión; la reflexión empieza..." (p.4). Con lo anterior se pretende mostrar que la práctica surge a partir del fracaso de las mismas, es decir, cuando el docente presenta desaciertos en su quehacer diario se ve obligado a reflexionar sobre ello, de tal forma que lo lleve a replantear lo realizado. De esta forma entiende que "la pedagogía no debe considerarse como la práctica pedagógica misma, sino como el saber teórico-práctico logrado por el docente a partir de la reflexión personal y dialogal sobre su propia práctica pedagógica” (Vasco, 2006, p. 2), buscando así mostrar que en la medida en que el docente entienda que la reflexión y una posición crítica frente a su práctica pedagógica son dos maneras de lograr una conciencia frente a las dificultades que se encuentran en el aula y solo cuando se realiza una crítica de manera propositiva (teniendo presente la investigación) sobre la misma se lograran mejores resultados cada día.

\section{La Reflexión a partir de la Enseñanza de las Ciencias}

Es importante recalcar que el docente en formación en ciencias, a partir de una reflexión continua, logrará que sus teorías sobre la enseñanza y aprendizaje cambien de manera significativa acorde a las teorías pedagógicas. Por ello es de gran importancia reflexionar a partir de los diferentes documentos que muestren los aportes que realicen los docentes que investigan en el campo de la enseñanza de las ciencias, de esta forma puede permitir a los docentes en formación experimentar y deliberar sobre las diversas dificultades en la enseñanza y aprendizaje. Generar espacios de reflexión en el aula 
de clase frente al análisis de documentos sobre investigaciones en la enseñanza de las ciencias, permite que los docentes en formación se fortalezcan desde lo disciplinar y lo pedagógico, como a su vez permite identificar posibles estrategias de enseñanza que contribuyan a superar dificultades de aprendizaje que ocurran en el aula de clase. Este tipo de ejercicios generará en los docentes en formación una reconstrucción conceptual desde el conocimiento disciplinar y pedagógico en marcado en el proceso de enseñanza y aprendizaje de las ciencias (Candela, 2012).

\section{La Reflexión sobre sí mismos, como Docentes en Formación}

Desde este punto de vista, los docentes en formación deben reflexionar sobre la comprensión y experiencia que poseen sobre los diferentes tópicos de la disciplina, lo cual contribuirá a dar significancia a las teorías sobre la enseñanza y aprendizaje que poseen. De esta forma les permitirá construir modelos teóricos que les facilitará plantear soluciones a las posibles dificultades que tengan frente a la disciplina.

Además de lo anterior los docentes en formación pueden reflexionar sobre su propia forma de aprender y las consecuencias de ellas al momento de planear la enseñanza de los diferentes tópicos de la disciplina, facilitando así el desarrollo de la categoría del conocimiento del contenido de la materia (Abell \& Bryan, 1997).

\section{Reflexionar sobre la Opinión de los Expertos}

Reflexionar sobre la posición de los diferentes expertos invita a los docentes en formación a acercarse y actualizarse sobre la bibliografía existente en investigaciones en el campo, como a su vez en las diferentes experiencias vividas. Este tipo de acercamiento es conveniente realizarlo bajo unas directrices claras, las cuales son dadas por el docente formador, para lo cual se pueden emplear instrumentos que le permitan abordar la lectura en varios momentos, un antes, durante y después de la misma. De esta forma se harán explícitos los conocimientos (antes de la lectura) mientras que el momento final de la lectura (después de la lectura) permitirá realizar asociaciones del contenido leído con el ya establecido en la estructura conceptual (Abell \& Bryan, citados por Candela, 2012).

Mecanismos como el mencionado anteriormente permiten que los docentes en formación piensen lo que ellos leen y la forma cómo estas se relacionan, tanto con las teorías personales ya construidas y con las otras experiencias recogidas en los diferentes cursos a los cuales ellos asistan. De esta forma los artículos o documentos que sean revisados por los docentes en formación no se considerarán como la máxima autoridad para aprender sobre la enseñanza y aprendizaje de una disciplina, pero se pueda considerar como un punto de partida para reevaluar y replantear las teorías personales (Abell \& Bryan, citados por Candela, 2012).

\section{Reflexionar sobre su propia Enseñanza}

Permitir que el docente en formación tenga la posibilidad de interactuar con sus pares enseñando tópicos de la disciplina, como a su vez el realizar su práctica en espacios formales (instituciones educativas) permitirá que interiorice su marco conceptual desde lo pedagógico y disciplinar (Abell \& Bryan, 1997). Para lograr que este tipo de experiencias formales sean significativas al docente en formación, es necesario tener un continuo acompañamiento por parte de los docentes formadores, facilitando así una continua reflexión (Candela, 2012). Se debe generar a partir del acompañamiento la reflexión teniendo presente la experiencia vivida, con el propósito de problematizar los juicios, pensamientos y acciones que los docentes en formación llevaron a cabo durante la experiencia vivida.

\section{La Mediación Didáctica en el Aula de Clase}

Folegotto (Citado por Pósito, 2012) expresa que una acción mediadora está ligada a buscar estrategias que permitan al estudiante reposicionarse frente al objeto de conocimiento, también plantean que la mediación didáctica son las acciones que realiza el docente, de tal forma que permita acompañar y promover el aprendizaje en los estudiantes; es tender puentes entre lo que el 
estudiante sabe y no sabe, entre lo vivido y lo que va a vivir, en otras palabras son puentes de relación e interacción, puentes terminológicos, técnicos, conceptuales.

Para trabajar en la medicación didáctica Pósito (2012) plantea la necesidad de tener en cuenta los siguientes aspectos al momento de realizarla:

- Tener en cuenta las características epistemológicas propias de las disciplinas del área en la medida en que ellas se convierten en el marco teórico y metodológico para estructurar los contenidos e identificarlos como conceptuales, procedimentales y actitudinales que el estudiante debe aprender.

- Centrar la atención en las diferentes actividades que se realizaran en el proceso al igual que los materiales didácticos a emplear y el cómo se emplearan.

- No basta con enunciar las actividades de aprendizaje a desarrollar dentro del proceso de mediación didáctica, estas deben tener una fundamentación y un sentido para lograr el aprendizaje, por ello se deben reconocer las capacidades y los procesos cognitivos involucrados, de tal forma que permitan seleccionar el método y recursos didáctico que favorezca la construcción del conocimiento científico escolar.

\section{Las Actividades De Aprendizaje Como Parte De La Mediación Didáctica}

Castillo (citado por Pósito, 2012) plantea la necesidad de tener presente que las actividades de aprendizaje representan el camino por los cuales transcurrirá la mediación didáctica. Una actividad de aprendizaje no se debe limitar a una mera acción, siendo así, la relación con el educando se estrechará, esta debe estar centrada en una explicación que permita dar sentido a la misma y con ello mostrar las consecuencias de su aprendizaje para así favorecer la apropiación del conocimiento. Dar sentido a la actividad de aprendizaje implica por parte del docente: dar explicaciones precisas sobre la actividad, establecer un dialogo con los estudiantes; tener presente los espacios y las situaciones que intervienen en el aprendizaje, plantearse interrogantes tales como: ¿con quién se aprende?, ¿con que se aprende?, ¿en qué momento se aprende? (instancias de aprendizaje); conocer las instancias de aprendizaje, tales como: la institución (concebida como el sistema en pleno: Materiales, instalaciones, compromisos, etc.); el educador (como la persona que orienta la mediación); los materiales didácticos (con ellos la mediación cobra su mayor sentido, pues son los recursos verbales, visuales, que facilitan el proceso); el grupo (el trabajo grupal facilita los aprendizajes si existe una previa planificación); el contexto (da un mayor sentido por parte de los estudiantes a la actividad misma); consigo mismo (se apropia el conocimiento en la medida en que se hace consciente del proceso).

\section{CONCLUSIÓN}

Es bien claro que los procesos de construcción del conocimiento científico escolar son complejos, pues son muchos los factores que influyen en esos procesos y es difícil mantener las condiciones ideales para lograr efectivamente dicha construcción, por ello cada vez el educador se enfrenta a una serie de retos que están encaminados a alcanzar los propósitos deseados y se ve obligado a tomar decisiones que buscan mejorar los procesos de enseñanza y aprendizaje a partir de su marco conceptual (disciplinar y pedagógico) y es este quien determina el papel que juega en dicho proceso, de ahí que un mediador en un proceso educativo se debe caracterizar por promocionar el aprendizaje de una manera creativa, abriendo espacios para la expresión en todos los ámbitos. Debe promover el aprendizaje, la construcción del conocimiento, las experiencias vividas dentro y fuera del aula de clase, de tal forma que le facilite la interiorización del conocimiento.

Actualmente el docente debe involucrarse cada día más en los procesos de mediación en el aula de clase, cuando el docente no lo hace, es decir, sustituye las experiencias de aprendizaje por contenidos, programas o curriculum por cumplir, lo que se logra es desvirtuar los procesos de mediación quedando inmerso en espacios que difícilmente generará en los estudiantes motivación alguna y deseos por aprender; por ello el 
docente debe lograr una conciliación entre los contenidos que se han programado en el currículo y las estrategias didácticas que se planteen para lograr una construcción del conocimiento, pues es bien claro la necesidad de cumplir con una planeación académica establecida para cada nivel de formación. Además de lo anterior el docente debe incentivar y persuadir a los estudiantes a partir de experiencias en las cuales los estudiantes se vean reflejados o involucrados de una forma $u$ otra, de esta forma el estudiante o sujeto que aprende, utiliza los sentidos que les permitirá acceder con mayor facilidad al conocimiento, siempre y cuando además de involucrar a los estudiantes a partir de experiencias se tenga en cuenta en gran medida la forma o estilo de aprendizaje.

Se ha encontrado que las experiencias que se plantean en el aula de clase son orientadas bajo la forma o estilo de aprendizaje de quien la orienta más no de quien será orientado, es decir se centra realmente en la forma como el docente aprende y no en el estudiante quien debe ser realmente el centro, es por ello que las actividades, estrategias, entre otras que se realicen como parte de los procesos de mediación didáctica se deben convertir en experiencias de aprendizajes gratas, placenteras y motivantes para los estudiantes, por lo tanto el énfasis de dichos procesos debe estar centrado en el acto de aprender para la vida, lo que implica una organización del conocimiento.

En la medida en que los docentes realicen su práctica teniendo como eje principal la reflexión continua frente a su quehacer diario, facilitara la construcción de un cumulo de conocimientos a partir de la solución de los diversos problemas que encontrara en su diario vivir (práctica); dicho conocimiento será el resultado de su experiencia como docente en el aula de clase, de esta forma construirá las herramientas que le permitirá ser más asertivo al momento de implementar las diversas estrategias, herramientas y metodologías que le conllevará a innovar en el aula de clase y así mismo facilitar la enseñanza y el aprendizaje en los estudiantes.

\section{REFERENCIAS BIBLIOGRÁFICAS}

Andréu, Abela Jaime (S.F). Las técnicas de Análisis de Contenido: Una revisión actualizada. Universidad de Granada.

Abell, K., \& Bryan, L. A. (1997). Reconceptualizing the Elementary Science Methods Course Using a Reflection Orientation. Journal of Science Teacher Education, Vol. 8 (3): 153-166.

Álvarez, A. Carmen. (2012). La relación teoría-práctica en los procesos de enseñanza-aprendizaje. Universidad de Cantabria. Educatio Siglo XXI, Vol. 30 (2): 383-402

Candela, B.F. (2012). La Captura, la Documentación y la Representación del CPC de un Profesor Experimentado y "ejemplar" Acerca del Núcleo Conceptual de la Discontinuidad de la Materia. Tesis de maestría. Universidad del Valle, Instituto de Educación y Pedagogía. Cali-Colombia.

Clark, C. \& Yinger, R.J. (1988). El uso de documentos personales en el estudio del pensamiento del profesor. En Villar Angulo L.M. (Dir.) (1988). Conocimiento, creencias y teorías de los profesores. Implicaciones para el curriculum y la formación del profesorado (pp. 175-195). Alcoy: Marfil.

Briceño, J., González, M., \& Mosquera, A. (2012). Estrategias de formación de docentes y estándares de competencias en TIC propuesta para Colombia. Computadores para educar. Ministerio de Tecnologías de la Información y las Comunicaciones. Bogotá: Colombia

Espinosa, R.E. (2009). Los mediadores pedagógicos en la enseñanza de las ciencias: la implementación de un programa educativo multimedia en la enseñanza del sistema circulatorio. El Hombre y la Máquina. Vol. 32: 20-36.

Fainholc, B. (2004). Lectura crítica en Internet. Análisis y utilización de los recursos tecnológicos en educación. Ediciones Homo Sapiens. Primera Edición. Rosario.

Gutiérrez, M., Ball, M. \& Marquez, E. (2007). Signo, Significado e Intersubjetividad: una Mirada Cultural. Educere Vol. 12 (43): 689-695. Hernández, G. (1997). Módulo Fundamentos del Desarrollo de la Tecnología Educativa (Bases Psicopedagógicas). Ediciones ILCE- OEA México. 
Korthagen, A. J. (2010). La práctica, la teoría y la persona en la formación del profesorado. Interuniversitaria de Formación del Profesorado. Vol. 68 (24,2): 83-101.

Lopez, B., \& Basto, S. (2010). Desde las teorías implícitas a la docencia como practica reflexiva. Educación y Educadores, Vol. 13 (2): 275-291.

Martínez, A. (2013). Emergencias de cambio: entre el modelo pedagógico tradicional y la necesidad de aprendizajes significativos. Praxis, 9, $73-82$

Mellado, V. (2003). Cambio Didáctico del Profesor de Ciencias Experimentales y Filosofía de la Ciencia. Enseñanza de las ciencias. Vol. 21 (3): 343-358.

Ministerio De Educación Nacional. (2008). Programa Nacional de Innovación Educativa con Uso de TIC. Programa estratégico para la competitividad. Ruta de apropiación de TIC en el Desarrollo Profesional Docente

Ministerio De Tecnologías De La Información Y Las Comunicaciones. (2012). La formación de docentes en TIC, casos exitosos de computadores para educar.

Perinat, A. (1986). La comunicación preverbal. Barcelona, España: Avesta S.A.

Pósito, R. M. (2012). El problema de enseñar $y$ aprender ciencias naturales en los nuevos ambientes educativos. Tesis de maestría, Universidad nacional de la plata.

Serrano, R. (2010). Pensamientos del profesor: un acercamiento a las creencias y concepciones sobre el proceso de enseñanza-aprendizaje en la educación superior. Revista de Educación Vol. 352: 267-287.

Seré, M. G. (2002). La enseñanza en el laboratorio. ¿Qué podemos aprender en términos de conocimiento práctico y de actitudes hacia la ciencia? Enseñanza de las ciencias Vol. 20 (3): 357-36.
Schön, D. A. (1987). La formación de profesionales reflexivos. Hacia un nuevo diseño de la enseñanza y el aprendizaje de las profesiones. Barcelona: Paidós.

Shavelson, R. \& Stern, P. (1989). Investigación sobre el pensamiento pedagógico del profesor, sus juicios, decisiones y conducta. En Gimeno J. \& Pérez Gómez, A. La enseñanza: su teoría y su práctica (pp. 372- 416). Madrid: Akal

Sisto, V. (1998). Del signo al sentido. Aproximaciones para un estudio semiótico de la conciencia [versión electrónica]. Santiago: Universidad de Arte y Ciencias Sociales. Recuperado el 10 de mayo de 2006 en http://168.96.200.17/ar/libros/chile/arcis/ sisto.rtf.

Tébar Belmonte Lorenzo (2009). El profesor mediador del aprendizaje. Bogotá, Colombia. Editorial magisterio.

Vaillant, D. (2013). Programa TIC y Educación Básica. Integración de TIC en los sistemas de formación docente inicial y continua para la Educación Básica en América Latina. Unicef primera edición. Argentina.

Vasco, C. E. (2006). Siete Retos de la Educación Colombiana para el periodo de 2006 a 2019. Universidad EAFIT. Medellín. Recuperado de http://www.eduteka.org/pdfdir/ RetosEducativos.pdf.

Vigotsky L. S. (1995). El desarrollo de los procesos psicológicos superiores. Barcelona:Crítica.

Wittrock, M. C. (1989). La investigación de la enseñanza I. Ediciones Paidos, $1^{a}$ edición. Madrid.

Zambrano, A. (2000). La relación entre el conocimiento del estudiante y el conocimiento del maestro en la educación en ciencias experimentales. Santiago de Cali, Universidad del Valle. Cali-Colombia. 